Ann. Génét. Sél. anim., I970, 2 (I), I0I-105.

\title{
HÉTÉROGÉNÉITÉ DES PROPORTIONS MENDÉLIENNES POUR 2 GÈNES DE GROUPES SANGUINS CHEZ LA POULE. DONNÉES COMPLÉMENTAIRES
}

\author{
A. PERRAMON et P. MÉRAT \\ avec la collaboration technique de J.-L. Monvoisin \\ Laboratoive de Génétique formelle, \\ Station centrale de Génétique animale, \\ Centre national de Recherches zootechniques, 78-Jouy-en-Josas \\ Institut national de la Recherche agronomique
}

\section{RÉSUMÉ}

Une hétérogénéité non aléatoire des proportions mendéliennes pour deux loci de groupes sanguins n'est observée que lorsque la mère est hétérozygote. Ceci renforce l'hypothèse précédemment émise d'une anomalie de ségrégation antérieure à la méiose.

Nous avons déjà signalé (Perramon et Mérat, Ig68), dans une souche de poules de race Wyandotte élevée au Domaine du Magneraud, la présence d'une hétérogénéité hautement significative des proportions mendéliennes entre mères au locus $W^{6} / W^{0}$, pour le croisement o $W^{0} / W^{0} \times q W^{6} / W^{0}$, et au locus $W^{7} / W^{8}$ lié au premier pour les croisements o $W^{7} / W^{7} \times q W^{7} / W^{8}$ et $\sigma^{7} W^{8} / W^{8} \times q W^{7} / W^{8}$ (au premier locus, $W^{0}$ indique l'absence de l'antigène identifié $W^{6}$; le second locus ne comporte que les deux allèles $W^{7}$ et $W^{8}$ dans la population étudiée).

Nous avions indiqué qu'un manque occasionnel de réactivité des sérums anti- $W^{6}$, anti- $W^{7}$ ou anti- $W^{8}$ n'était pas vraisemblable, la proportion globale pour chacun des produits des deux loci dans l'ensemble des familles étant sensiblement normale. Une différence de mortalité embryonnaire associée à ces antigènes devrait être, pour expliquer nos résultats, de sens variable suivant les familles; de toutes façons, les taux d'éclosion sont aussi bons pour les mères à 


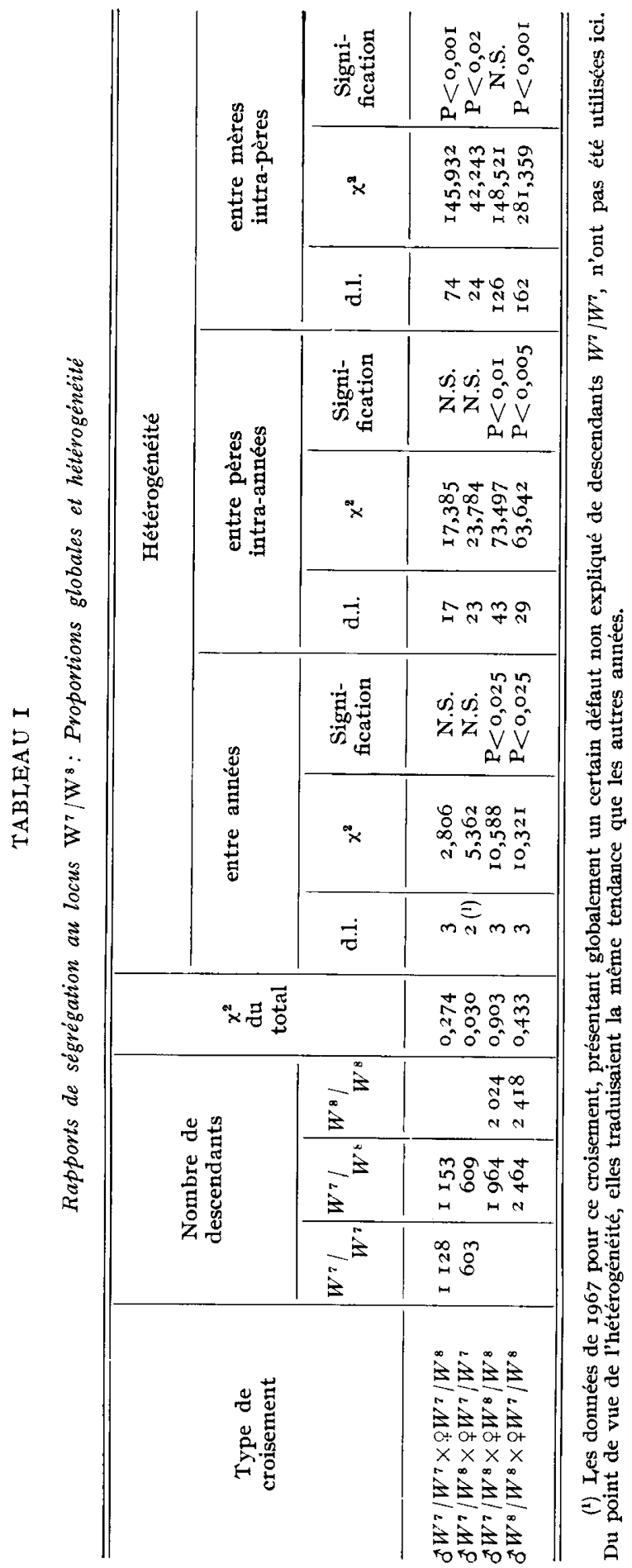


proportion anormale que pour les autres. Ceci suggère des influences non aléatoires sur les proportions mendéliennes, se situant avant la méiose de la femelle.

L,es résultats précédents portaient sur les années I964, I965 et r966.

En 1967 et I968, nous avons pu réunir des données complémentaires relativement au locus $W^{7} / W^{8}$. Ces données paraissent confirmer l'absence de relation entre le rapport de ségrégation d'une mère hétérozygote à ce locus et son taux de fertilité et d'éclosion; mais surtout, l'analyse plus complète de 1'hétérogénéité des proportions qu'elles permettent apporte des précisions renforçant indirectement nos précédentes conclusions.

Pour l'ensemble des années, y compris les dernières, le tableau I donne pour chaque type de croisement étudié au locus $W^{7} / W^{8}$ la proportion totale des descendants des deux types attendus, sexes groupés, puis successivement les $\chi^{2}$ d'hétérogénéité de cette proportion entre années, entre pères intra-années et entre mères intra-pères (cf. MATHER, r96o).

La comparaison des croisements réciproques suggère à première vue une hétérogénéité plus grande entre mères intra-pères lorsque la mère est hétérozygote. C'est ce que l'on vérifie de façon plus précise en totalisant, d'une part les deux croisements où c'est le père, de l'autre ceux où c'est la mère qui est de génotype $W^{7} / W^{8}$. Dans le premier cas, le $\chi^{2}$ d'hétérogénéité "mères intra-pères " vaut I90,764 pour I50 degrés de liberté; dans le second, 427,29I pour 236 degrés de liberté.

Si l'on fait dans chaque cas le rapport du $\chi^{2}$ à son nombre de degrés de liberté, on obtient : $s^{2}{ }_{1}=\mathrm{I}, 27 \mathrm{I}$ lorsque le père est hétérozygote, et : $s^{2}{ }_{2}=\mathrm{r}, 8 \mathrm{Ir}$ lorsque c'est la mère. Dans l'hypothèse de l'égalité des "valeurs vraies" de ces deux variables, le rapport $s_{2}{ }_{2} / s^{2}{ }_{3}$ doit suivre une loi de $F$ (Fisher-Snedecor) à 236 et $\mathbf{I}_{50}$ degrés de liberté. La valeur de ce rapport étant $I, 425$, on vérifie qu'elle est significativement supérieure à l'unité au seuil approximatif de I p. Ioo.

Par contre, en ce qui concerne l'hétérogénéité entre pères, elle ne paraît pas différer nettement suivant le caractère homozygote ou hétérozygote du génotype paternel. En fait, étant donnée l'existence d'une hétérogénéité très significative des proportions entre mères, on doit prendre en considération, non le $\chi^{2}$ d'hétérogénéité entre pères lui-même, mais le rapport de la "variance entre pères " ( $\chi^{2}$ " entre pères " divisé par le nombre de degrés de liberté correspondant) à la "variance " analogue "entre mères ". Pour les croisements où le père est homozygote, ce rapport, que l'on peut considérer comme suivant approximativement une loi de $\mathrm{F}$, est égal à 0,97 ; sa valeur est $\mathrm{I}, \mathrm{I} 6$ lorsque le père est hétérozygote. Dans les deux cas, il n'apparaît pas d'hétérogénéité significative attribuable en propre au père.

Il en est de même de 1'hétérogénéité entre années : en la divisant par le nombre de degrés de liberté qui lui correspond, on obtient une "variance entre années " égale à 2,048 pour l'ensemble des croisements, basée sur ${ }_{5} 5$ degrés de liberté. Son rapport à la variance "entre mères intra-pères ", basée sur $5^{02}$ degrés de liberté au total, est $F=r, 29 I$ (non significatif).

Quant au locus $W^{6} / W^{0}$, seule l'année I $^{6} 67$ a permis d'apporter en ce qui le concerne des résultats nouveaux. L'ensemble de nos données actuelles indique 
pour lui la même tendance : le $\chi^{2}$ d'hétérogénéité "entre mères intra-pères " est, au total, égal à 62,994 pour 27 degrés de liberté $(\mathrm{P}<0,00 \mathrm{I})$ dans les croisements $\sigma^{*} W^{0} / W^{0} \times{ }^{0} W^{6} / W^{0}$ où la mère est hétérozygote, sa valeur étant 65 , I 90 pour 57 d.1. $(\mathrm{P}>0,2)$ dans les croisements of $W^{6} / W^{0} \times W^{0} / W^{0}$. Le rapport $\mathrm{F}$ des deux $\chi^{2}$ divisés chacun par le nombre de degrés de liberté correspondant est 2,04I $(P<0,025)$.

Au total, le fait saillant est donc l'existence d'une hétérogénéité très prononcée des proportions mendéliennes, essentiellement lorsque la mère est hétérozygote aux loci de groupes sanguins considérés. Ceci renforce notre hypothèse quant à la réalité d'une variation non aléatoire de ces proportions au niveau des gamètes, car on comprendrait difficilement qu'une pénétrance incomplètẹ d'un allèle ou une différence de mortalité embryonnaire se manifeste seulement par des différences entre descendances de mères hétérozygotes. Notre interprétation par une anomalie de ségrégation ayant sa source à la méiose ou avant celle-ci (PERRAmon et MÉRAT, I968) se trouve ainsi, indirectement, appuyée. Quant à la nature de cette anomalie, l'expulsion préférentielle d'un allèle dans un globule polaire doit être écartée parce qu'en contradiction avec les données de linkage exposées précédemment (PERramon et MÉRAT, Ig68). De même, l'existence d'un mécanisme de conversion génique ne paraît pas convenir car, ayant été décrite comme une éventualité essentiellement méiotique, elle devrait s'être produite pendant plusieurs méioses successives. Il reste donc la possibilité d'un crossing-over "mitotique " (STERN, I936; PONTECORVo, I958) ou de tout mécanisme analogue tendant à un mosaïcisme des gonades. Des conclusions similaires ont été suggérées chez d'autres vertébrés supérieurs (GRUNEBERG, I966; BEÇAK et al., I966).

Reçu pour publication en novembre 1969.

\title{
REMERCIEMENTS
}

Nous remercions le professeur Brun, Faculté des Sciences, Orsay, et M. Grosclavde, Laboratoire de Génétique biochimique, C.N.R.Z., Jouy-en-Josas, de leurs remarques à la lecture de ce manuscrit.

\section{SUMMARY}

\author{
HETEROGENEITY OF MENDELIAN PROPORTIONS \\ FOR TWO BLOOD-GROUP GENES IN THE FOWL. FURTHER DATA
}

A non-random heterogeneity of mendelian proportions was observed between progenies of different dams in the segregation to two blood-group loci in a White Wyandotte strain. One of the loci has two alleles, $W^{7}$ and $W^{8}$; for the other, linked to the first one, an allele, $W^{6}$, has been identified, $W^{0}$ representing its absence. The present data, which complete a previous work, conffrm more precisely that this phenomenon is limited to the case when the dam is heterozygous. The "between dams " heterogeneity is highly significant $(P<.001)$ in this case. This supports further our hypothesis of a segregation abnormality taking place at or before meiosis and tending to cause a mosaicism of gonadal tissue. 


\section{RÉFÉRENCES BIBLIOGRAPHIQUES}

BEÇAK W., BEÇAK M.L., OHNo S., r966. Intra individual chromosomal polymorphism in green Sunfish (Lepomis cyanellus) as evidence of somatic segregation. Cytogenetics, 5, 313-320.

GRUNEBERG H., r966. The case for somatic crossing-over in the Mouse. Genet. Res., 7, 58-75.

Howaro A., Mc LAREN A., Mrchie D., SANDer G., 1955. Genetic and environmental influences on the secondary sex ratio in Mice. J. Genet., 53, 200-214.

MATHER K., r96o. Statistical analysis in biology. Methuen \& Co, I,ondon, $4^{\mathrm{e}}$ éd.

Perramon A., Merat P., r968. Étude génétique des groupes sanguins dans deux populations aviaires. I. Transmission héréditaire des facteurs antigéniques. Ann. Biol. anim. Bioch. Biophys., 8, 485+50o. Ponteconvo G., I958. Trends in genetic analysis. Columbia University Press, New York.

STERN C., 1936. Somatic crossing -over and segregation in Drosophila melanogaster. Genetics, 21, 625-730. 